\title{
Translational research in anti- pancreatic fibrosis drug discovery and development
}

\author{
Xingyu Ze ${ }^{1,2 \#}$, Wenbin Zou ${ }^{1,2 \#}$, Zhaoshen $\mathrm{Li}^{1,2}$ \\ 'Department of Gastroenterology, Digestive Endoscopy Center, Changhai Hospital, \\ Naval Medical University, Shanghai 200433, China; \\ ${ }^{2}$ Shanghai Institute of Pancreatic Diseases, Shanghai 200433, China
}

\#These authors contributed equally to this paper.

Address for Correspondence: Dr. Zhaoshen Li, Department of Gastroenterology, Changhai Hospital, Naval Medical University, 168 Changhai Rd, Yangpu District, Shanghai, China; Shanghai Institute of Pancreatic Diseases, 168 Changhai Road, Shanghai 200433, China

E-mail: zhaoshenli@smmu.edu.cn

Access this article online

Website:

www.intern-med.com

DOI:

10.2478/jtim-2021-0036

Quick Response Code:

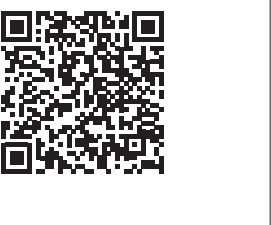

Pancreatic fibrosis is a characteristic pathological feature of chronic pancreatitis (CP). It is closely related to the activation of pancreatic stellate cells (PSCs) resulting in the secretion of excess extracellular matrix (ECM) proteins such as collagen, $\alpha$-smooth muscle actin ( $\alpha$-SMA) and fibronectin. $\mathrm{CP}$ is a progressive inflammatory disease of the pancreatic tissue accompanied by pancreatic exocrine and endocrine insufficiency. Alcohol, hereditory factors, the environmental and other risk factors can lead to $\mathrm{CP} ;{ }^{[1]}$ these risk factors can also cause pancreatic fibrosis. Gene mutation has been a focus of research on the progression of CP in recent years; mutations that have received research attention include protease serine 1 (PRSS1, a point mutation of the cationic trypsinogen gene), serine protease inhibitor Kazal type 1 (SPINK1), chymotrypsin C (CTRC), and cystic fibrosis transmembrane conductance regulator (CFTR). ${ }^{[2]}$ The pathogenesis of pancreatic fibrosis is complex and not completely understood.

Activated PSCs play a key role in pancreatic fibrosis. PSCs are desmin- and glial fibrillary acidic protein (GFAP)-positive cells around pancreatic acini, blood vessels, and ducts; in 1998, they were first successfully separated and cultured, similar to hepatic stellate cells, from the rat pancreas and resected human pancreatic tissues, respectively. ${ }^{[3-5]}$ PSCs can be activated by pro-inflammatory cytokines [tumor necrosis factor (TNF)- $\alpha$, interleukin (IL) $-1 \beta$, IL-4, etc.] and chemokines [chemokine (C-C motif) ligand (CCL)s and chemokine (C-X-C motif) ligand (CXCLs)]. Meanwhile, it has been demonstrated that signaling pathways such as the transforming growth factor- $\beta$ (TGF- $\beta$ )/small mothers against decapentaplegic (Smad) ${ }^{[6]}$ wingless/ integrated (WNT), ${ }^{[7]}$ mitogen-activated protein kinase (MAPK), ${ }^{[8]}$ Janus-activated kinase (JAK)/signal transducer and activator of transcription (STAT), ${ }^{[9]}$ and Notch ${ }^{[10]}$ pathways are closely related to the fibrosis process. Macrophages also interact with PSCs in an autocrine and paracrine cytokine-dependent manner in CP (M2 polarization). ${ }^{[11]}$ All of these factors lead to ECM deposition in the pancreas and stimulate pancreatic fibrosis, even if the pathogeny is removed. In recent years, several studies have shown that small molecular drugs and herbal extracts appear to have effects on pancreatic fibrosis.

Drugs from small molecules are among the most marketed therapeutics and have several advantages including well-defined structures, ease of manufacturing, and non-immunogenic properties. Researchers have attempted to identify effective antipancreatic fibrosis drugs, but, to date, none are widely used in clinical practice. Discoidin domain receptor 1 (DDR1) is the primary mediator of the dialog between the intracellular and ECM environment and plays a major role in epithelial/mesenchymal crosstalk in the mouse model; thus, it is one of the most attractive targets for fibrosis. ${ }^{[12]}$ However, the biology of DDR1 remains largely unknown. Dasatinib is a smallmolecule DDR1 inhibitor that has been found to have an anti-pancreatic fibrosis effect. In our institute, we found that dasatinib, an oral medicine for the treatment of certain types of chronic myeloid leukemia, could ameliorate CP through both antifibrotic and anti-inflammatory 
mechanisms in PSCs and a cerulein-induced CP mouse model. ${ }^{[13]}$ Dasatinib inhibited the proliferation and activation of PSCs and increased glycogen synthase kinase (GSK) $3 \beta$-mediated $\beta$-catenin cytosol retention by inhibiting multiple tyrosine kinases (TKs) and MAPK cascades. It also restrained M1 and M2 polarization of macrophages and impeded their recruitment and crosstalk with PSCs. ${ }^{[13]}$ Dasatinib may be a potential therapeutic agent for the treatment of patients with CP in the future. Camostat mesilate, an orally administered low-molecular-weight serine protease inhibitor, can effectively inhibit trypsin, plasma kallikrein, plasmin, and thrombin; this drug has long been used in clinical practice in Japan for the treatment of $\mathrm{CP}$, but its efficacy remains uncertain. A previous study reported that camostat mesilate can also suppress the gene expression of pancreatitis-associated protein (PAP), p8 (a gene activated in the pancreas during the acute phase of pancreatitis), IL-6, and TGF- $\beta$; thus, it may be used to treat for pancreatic fibrosis. ${ }^{[14]}$ Unfortunately, to date, there is no evidence as to whether camostat mesilate can improve the long-term prognosis of patients with CP. Nonetheless, if patients take this medicine at the early stage of $\mathrm{CP}$, there is evidence suggesting that it could inhibit pancreatic inflammation and pancreatic fibrosis. ${ }^{[15]}$ In a randomized placebo-controlled trial, patients with $\mathrm{CP}$ were given antioxidant supplementation including daily doses of 600 $\mu \mathrm{g}$ of organic selenium, $0.54 \mathrm{~g}$ of vitamin C, $9000 \mathrm{IU}$ of $\beta$-carotene, $270 \mathrm{IU}$ of vitamin $\mathrm{E}$, and $2 \mathrm{~g}$ of methionine for 3 months. Pancreatic fibrosis markers, including serum levels of TGF- $\beta 1$ and platelet-derived growth factor-AA, were significantly reduced compared to the placebo group. ${ }^{[16]}$ However, the real effects of the above-mentioned drugs must be evaluated in high-quality clinical studies with longterm follow-ups and examination of pathological changes in pancreatic tissue to prove their efficacy.

There is a long history of use of herbal medicines in China and other Asian countries. Herbal drugs are very cheap in comparison to conventional forms of medication and can mostly be accessed without a prescription. For instance, compound Danshen dripping pills are widely used in coronary heart disease in China for their pain relief, invigoration, and blood circulation effects. There are hundreds of thousands of herbal drugs in use all over the world. Emerging evidence suggests that some of these herbal drugs could provide a novel approach to anti-pancreatic fibrosis. A study from Japan showed that Saiko-Keishi-to (TJ-10), an herbal drug, exhibited antipancreatic fibrosis effect in WBN/Kob rats. ${ }^{[17]} \mathrm{TJ}-10$ is an herbal derivative with anti-inflammatory, analgesic, antiemetic, and immunomodulatory properties; it is a mixture of extracts from different medical herbs with different pharmacologic actions. TJ-10 has been shown to suppress pancreatic fibrosis through suppression of TGF- $\beta 1$ and
PAP gene expression in WBN/Kob rats. ${ }^{[17,18]}$ Baicalei, as a member of TJ-9, has been shown to inhibit the activation of PSCs. ${ }^{[19]}$ However, current studies are limited to rat CP models; there is little published clinical data.

A study from Korea reported that DA-9601 had antipancreatic fibrosis effect in a CP mouse model. ${ }^{[20]} \mathrm{DA}-9601$ is an antioxidative phytochemical derived from the ethanol extract of Artemisia asiatica; it has long been used in folk medicine as an adjuvant therapy for several inflammatory diseases. CP mice treated with DA-9601 had lower levels of inducible nitric oxide synthase and myeloperoxidase, higher levels of cytoprotective proteins such as heat shock protein-70 and metallothionein, and decreased expression of $\alpha$-SMA and collagen I in cultured PSCs. Therefore, the authors concluded that this substance has antioxidative, anti-inflammatory, and cytoprotective properties. Another herbal drug, berberine, is a quaternary ammonium alkaloid isolated from Chinese rhizomacoptidis, cortex phellodendri, and berberis plants. It has been used both in China and Western countries for over 30 years as a clinical medicine for intestinal infection and diarrhea. ${ }^{[21]}$ A systematic literature review showed that it can also reduce blood glucose levels in patients with type 2 diabetes mellitus. ${ }^{[22]}$ Further, a study from India showed that berberine acts as an AMP-activated protein kinase (AMPK) activator and may have antioxidant and anti-inflammatory effects. ${ }^{[23]}$ In this study, the researchers observed the effects of berberine against CP in cerulein-induced mice and reported that it acted by inhibiting TGF- $\beta /$ Smad signaling and M2 macrophage polarization. ${ }^{[23]}$ Another study from China showed that the application of phenolic compounds significantly suppressed the mRNA and protein levels of $\alpha$-SMA, collagen I, and fibronectin and activated the nuclear factor-kappa B signaling pathway in large $T$ immortalized cells (LTC)-14 cells against TGF- $\beta$ stimulation. ${ }^{[24}$ Although herbal drugs have good prospects, they have complex ingredients with multiple targets and pathways and are still not widely accepted by most researchers.

Anti-organ fibrosis has been a difficult problem for clinicians for many years. While there are many published studies on the mechanisms of fibrosis and there has been great progression in this field, research on pancreatic fibrosis has remained stagnant in recent years. There is still a lack of availability of clinical drugs that have definite effects on degrading ECM to cure pancreatic fibrosis. Moreover, there are no randomized controlled trials in humans. Nonetheless, the agents discussed above have definitely paved the way for the evolution of a logical and scientific approach to the study and management of pancreatic fibrosis. At present, mitigation of risk factors, correct and early diagnosis, and early medical intervention are the best ways to delay the progression of $\mathrm{CP}$ and 
pancreatic fibrosis. ${ }^{[25]}$ From the studies described above, it is clear that there are several drugs that have potential anti-pancreatic fibrosis effects. Unfortunately, there are not yet any therapeutic drugs that are widely acknowledged and have definite efficacy on pancreatic fibrosis; thus, it is necessary to further evaluate the effects of these drugs in the future to complete the process of translational medicine and take these drugs to the next step.

\section{Source of Funding}

The work is supported by the National Natural Science Foundation of China (81873588 [Z.S.L.], 82070661 [W.B.Z.])

\section{Conflict of Interests}

None declared.

\section{REFERENCES}

1 Whitcomb DC, Frulloni L, Garg P, Greer JB, Schneider A, Yadav D, et al. Chronic pancreatitis: An international draft consensus proposal for a new mechanistic definition. Pancreatology 2016;16:218-24.

2 Zou WB, Tang XY, Zhou DZ, Qian YY, Hu LH, Yu FF, et al. SPINK1, PRSS1, CTRC, and CFTR Genotypes Influence Disease Onset and Clinical Outcomes in Chronic Pancreatitis. Clin Transl Gastroenterol 2018; 9: 204-15.

3 Ito, T. Cytological studies on stellate cells of Kupffer and fat storing cells in the capillary wall of the human liver. Acta Anat. Nippon 1951;26:42 (in Japanese).

4 Apte MV, Haber PS, Applegate TL, Norton ID, McCaughan GW, Korsten MA, et al. Periacinar stellate shaped cells in rat pancreas: identification, isolation, and culture. Gut 1998; 43:128-33.

5 Bachem MG, Schneider E, Gross H, Weidenbach H, Schmid RM, Menke A, et al. Identification, culture, and characterization of pancreatic stellate cells in rats and humans. Gastroenterology 1998;115:421-32.

6 Dituri F, Cossu C, Mancarella S, Giannelli G. The Interactivity between TGF $\beta$ and BMP Signaling in Organogenesis, Fibrosis, and Cancer. Cells. 2019; 8:1130.

7 Piersma B, Bank RA, Boersema M. Signaling in Fibrosis: TGF- $\beta$, WNT, and YAP/TAZ Converge. Front Med (Lausanne). 2015; 2: 59.

8 Kyriakis JM, Avruch J. Mammalian MAPK signal transduction pathways activated by stress and inflammation: a 10-year update. Physiol Rev 2012; 92: 689-737.

9 Komar HM, Serpa G, Kerscher C, Schwoegl E, Mace TA, Jin M, et al. Inhibition of Jak/STAT signaling reduces the activation of pancreatic stellate cells in vitro and limits caerulein-induced chronic pancreatitis in vivo. Sci Rep 2017;7:1787-96.

$10 \mathrm{Hu}$ B, Phan SH. Notch in fibrosis and as a target of anti-fibrotic therapy. Pharmacol Res 2016; 108:57-64.

11 Gukovskaya AS, Gukovsky I, Algül H, Habtezion A. Autophagy, Inflammation, and Immune Dysfunction in the Pathogenesis of Pancreatitis. Gastroenterology 2017;153: 1212-26.

12 Cooley MM, Thomas D, Deans K, Peng Y, Lugea A, Pandol SJ, et al. Deficient Endoplasmic Reticulum Acetyl-CoA Import in Pancreatic Acinar Cells Leads to Chronic Pancreatitis. Cell Mol Gastroenterol Hepatol 2021;11:725-38.

13 Zeng XP, Wang LJ, Guo HL, He L, Bi YW, Xu ZL, et al. Dasatinib ameliorates chronic pancreatitis induced by caerulein via anti-fibrotic and anti-inflammatory mechanism. Pharmacol Res 2019;147:104357.

14 Su SB, Motoo Y, Iovanna JL, Xie MJ, Sawabu N. Effect of camostat mesilate on the expression of pancreatitis-associated protein (PAP), p8, and cytokines in rat spontaneous chronic pancreatitis. Pancreas 2001;23:134-40.

15 Ito $\mathrm{T}$, Ishiguro $\mathrm{H}$, Ohara $\mathrm{H}$, Kamisawa $\mathrm{T}$, Sakagami J, Sata N, et al. Evidence-based clinical practice guidelines for chronic pancreatitis 2015. J Gastroenterol 2016; 51: 85-92.

16 Dhingra R, Singh N, Sachdev V, Upadhyay AD, Saraya A. Effect of antioxidant supplementation on surrogate markers of fibrosis in chronic pancreatitis: a randomized, placebo-controlled trial. Pancreas 2013;42: 589-95.

17 Su SB, Motoo Y, Xie MJ, Taga H, Sawabu N. Antifibrotic effect of the herbal medicine Saiko-keishi-to (TJ-10) on chronic pancreatitis in the WBN/Kob rat. Pancreas 2001;22: 8-17.

18 Motoo Y, Su SB, Xie MJ, Taga H, Sawabu N. Effect of herbal medicine Saiko-keishi-to (TJ-10) on rat spontaneous chronic pancreatitis: comparison with other herbal medicines. Int J Pancreatol 2000; 27:123-9.

19 Sakaida I, Matsumura Y, Akiyama S, Hayashi K, Ishige A, Okita K. Herbal medicine Sho-saiko-to (TJ-9) prevents liver fibrosis and enzyme-altered lesions in rat liver cirrhosis induced by a choline-deficient $\mathrm{L}$-amino aciddefined diet. J Hepatol 1998;28: 298-306.

20 Yoo BM, Oh TY, Kim YB, Yeo M, Lee JS, Surh YJ, et al. Novel antioxidant ameliorates the fibrosis and inflammation of cerulein-induced chronic pancreatitis in a mouse model. Pancreatology 2005;5:165-76.

21 Yu M, Jin X, Liang C, Bu F, Pan D, He Q, et al. Berberine for diarrhea in children and adults: a systematic review and meta-analysis. Therap Adv Gastroenterol 2020;13: 1756284820961299.

22 Liang Y, Xu X, Yin M, Zhang Y, Huang L, Chen R et al. Effects of berberine on blood glucose in patients with type 2 diabetes mellitus: a systematic literature review and a meta-analysis. Endocr J 2019; 66: 51-63.

23 Bansod S, Doijad N, Godugu C. Berberine attenuates severity of chronic pancreatitis and fibrosis via AMPK-mediated inhibition of TGF- $\beta 1 / \mathrm{Smad}$ signaling and M2 polarization. Toxicol Appl Pharmacol 2020;403:165-76.

24 Lin Z, Zheng LC, Zhang HJ, Tsang SW, Bian ZX. Anti-fibrotic effects of phenolic compounds on pancreatic stellate cells. BMC Complement Altern Med 2015;15: 259-65.

25 Hart PA, Conwell DL. Chronic Pancreatitis: Managing a Difficult Disease. Am J Gastroenterol 2020;115:49-55.

How to cite this article: Ze X, Zou W, Li Z. Translational research in anti-pancreatic fibrosis drug discovery and development. J Transl Intern Med 2021; 9: 225-7. 